\title{
Regularization method for Noor's variational inequality problem induced by a hemicontinuous monotone operator
}

\author{
Yeol Je Cho ${ }^{1}$ and Narin Petrot ${ }^{2,3^{*}}$
}

"Correspondence: narinp@nu.ac.th

2Department of Mathematics,

Faculty of Science, Naresuan

University, Phitsanulok, 65000,

Thailand

${ }^{3}$ The Centre of Excellence in

Mathematics, CHE, Si Ayuthaya

Road, Bangkok, 10400, Thailand

Full list of author information is

available at the end of the article

\begin{abstract}
In this paper, we provide a regularization method for finding a solution of Noor's variational inequality problem induced by a hemicontinuous monotone operator. Moreover, such a solution is related to the set of zero of inverse strongly monotone mappings. Consequently, since we do not assume the strong monotonicity of the considered operator, our results are general and extend some well-known results in the literature.
\end{abstract}

Keywords: general variational inequality problem; regularization; hemicontinuous mapping; monotone mapping; inverse strongly monotone mapping

\section{Introduction and preliminaries}

It is well known that the variational inequalities theory, which was introduced by Hartman and Stampacchia [1] in early 1960s, provides the most natural, direct, simple, unified and efficient framework for a general treatment of a wide class of linear and nonlinear problems. Of course, it has been extended and generalized in several directions.

Let $H$ be a real Hilbert space with inner product $\langle\cdot, \cdot\rangle$ and norm $\|\cdot\|$. An important and useful generalization of variational inequality problem is called the general variational inequality introduced by Noor [2] in 1988, which is a problem of finding $u^{*} \in H$ with $g\left(u^{*}\right) \in K$ such that

$$
\left\langle A\left(u^{*}\right), g(v)-g\left(u^{*}\right)\right\rangle \geq 0
$$

for all $v \in H$ with $g(v) \in K$, where $K$ is a nonempty closed convex subset of $H$ and $A, g$ : $H \rightarrow H$ are mappings.

It is known that a class of nonsymmetric and odd-order obstacle, unilateral and moving boundary value problems arising in pure and applied sciences can be studied in the unified framework of general variational inequality (1.1), see [3-12] and the references therein. However, it is worth knowing that to guarantee the existence and uniqueness of a solution of the problem (1.1), one has to impose some conditions on the operator $A$ and $g$ (see [13] for an example in a more general case). Indeed, it has been shown that if $A$ fails to be Lipschitz continuous or strongly monotone, then the solution set of the problem (1.1), which will be denoted by $\operatorname{GVI}_{K}(A, g)$, may be empty.

(C) 2012 Cho and Petrot; licensee Springer. This is an Open Access article distributed under the terms of the Creative Commons Attribution License (http://creativecommons.org/licenses/by/2.0), which permits unrestricted use, distribution, and reproduction in any medium, provided the original work is properly cited. 
On the other hand, for a nonempty subset $K$ of $H$, recall that a mapping $T: K \rightarrow H$ is said to be nonexpansive if

$$
\|T x-T y\| \leq\|x-y\|
$$

for all $x, y \in K$. For a mapping $T: K \rightarrow H$, an element $x \in K$ is called a fixed point of $T$ if $x=T x$. Here, we will denote the set of all fixed points of the mapping $T$ by $F(T)$. It is well known that the problem of finding the fixed point sets for the class of nonexpansive mappings is the subject of current interest in nonlinear functional analysis. A large number of mathematicians are interested in this field, one may see some important results in [14-17]. Moreover, it is well known that there are some relations between the fixed point problems and many types of variational inequality problems. Hence, of course, it is natural to consider a unified approach to these two different problems (see [13, 18-21] for examples).

A mapping $A: K \rightarrow H$ is said to be $\lambda$-inverse strongly monotone if there exists $\lambda>0$ such that

$$
\langle A(x)-A(y), x-y\rangle \geq \lambda\|A(x)-A(y)\|^{2}
$$

for all $x, y \in K$.

For a mapping $A: K \rightarrow H$, we put $S_{A}=\{x \in K: A(x)=0\}$. Further, if $x \in S_{A}$, then $x$ is called a zero of $A$.

Remark 1.1 It is easy to see that any 1-inverse strongly monotone mapping is a nonexpansive mapping. Moreover, if $T$ is a nonexpansive mapping, then it is well known that $(I-T)$ is $\frac{1}{2}$-inverse strongly monotone mapping (see $\left.[22,23]\right)$. Also, let us notice that the problem of finding an element of $F(T)$ is equivalent to the problem of finding an element of $x \in S_{(I-T)}$. These mean that the problem of finding zeros of the inverse strongly monotone mappings contains the problem of finding fixed points of nonexpansive mappings as a special case.

Let $A: H \rightarrow H$ be a mapping and $A_{i}: K \rightarrow H$ be $\lambda_{i}$-inverse strongly monotone mapping for each $i=1,2, \ldots, N$. In this paper, we present a method for finding a solution of the problem (1.1), which is related to the solution sets of those inverse strongly monotone mappings as follows: find $u^{*} \in H$ with $g\left(u^{*}\right) \in S$ such that

$$
\left\langle A\left(u^{*}\right), g(v)-g\left(u^{*}\right)\right\rangle \geq 0
$$

for all $v \in H$ with $g(v) \in K$, where $S=\bigcap_{i=1}^{N} S_{A_{i}}$. Further, $\operatorname{GVI}_{K}(A, g, S)$ denotes a set of solutions of the problem (1.2).

\section{Remark 1.2}

(1) If $A_{i}=: 0$ (: the zero operator) for all $i=1,2, \ldots, N$, then the problem (1.2) reduces to $(1.1)$.

(2) If $g=: I$ (: the identity operator), then the problem (1.2) is reduced to the problem of finding the common element of the solution of classical variational inequality 
problem and the set $S$, which has been studied by many authors (see [23-25] for examples).

We need the following concepts in order to prove our results.

Throughout this paper, we let $\mathcal{R}$ and $\mathcal{N}$ stand for the set of real numbers and the set of natural numbers, respectively. Let $F: K \times K \rightarrow \mathcal{R}$ be a bifunction with $F(u, u)=0$ for all $u \in K$.

Definition 1.3 A bifunction $F: K \times K \rightarrow \mathcal{R}$ is said to be:

(1) monotone if, for all $u, v \in K$,

$$
F(u, v)+F(v, u) \leq 0
$$

(2) strongly monotone with constant $\tau$ if, for all $u, v \in K$,

$$
F(u, v)+F(v, u) \leq-\tau\|u-v\|^{2}
$$

(3) hemicontinuous in the first variable $u$ if, for any fixed $v \in K$,

$$
\lim _{t \rightarrow+0} F(u+t(z-u), v)=F(u, v)
$$

for all $(u, z) \in K \times K$.

Recall that the equilibrium problem for a bifunction $F: K \times K \rightarrow \mathcal{R}$ is to find $u^{\prime \prime} \in K$ such that

$$
F\left(u^{*}, v\right) \geq 0
$$

for all $v \in K$.

Considering the problem (1.3), the following are very useful.

Lemma 1.4 [26] Let $F: K \times K \rightarrow \mathcal{R}$ be a bifunction. If $F(u, v)$ is convex and lower semicontinuous in the variable $v$ for any fixed $u \in K$, then we have the following:

(1) If $F(u, v)$ is hemicontinuous in the first variable and has the monotonic property, then $U^{*}=V^{*}$, where $U^{*}$ is the solution set of $(1.3), V^{*}$ is the solution set of $F\left(u, v^{*}\right) \leq 0$ for all $u \in K$. Moreover, in this case, they are closed and convex;

(2) If $F(u, v)$ is hemicontinuous in the first variable for each $v \in K$ and $F$ is strongly monotone, then $U^{*}$ is a nonempty singleton. In addition, if $F$ is a strongly monotone mapping, then $U^{*}=V^{*}$ is a singleton set.

In order to prove our results, we also need the following concepts and facts.

Definition 1.5 A mapping $A: H \rightarrow H$ is said to be hemicontinuous at a point $x \in H$ if

$$
\lim _{t \rightarrow 0}\langle A(x+t h), y\rangle=\langle A(x), y\rangle
$$

for all $h, y \in H$. 
Definition 1.6 Let $H$ be a real Hilbert space and $K$ be a subset of $H$. A mapping $T: K \rightarrow H$ is said to be $k$-strictly pseudocontrative if there exists a constant $k \in[0,1)$ such that

$$
\|T x-T y\|^{2} \leq\|x-y\|^{2}+k\|(I-T)(x)-(I-T)(y)\|^{2}
$$

for all $x, y \in K$, where $I$ is the identity operator on $K$.

Remark 1.7 It is well known that if $T: K \rightarrow H$ is a $k$-strictly pseudocontrative mapping, then the mapping $A:=I-T$ is a $\left(\frac{1-k}{2}\right)$-inverse strongly monotone. Conversely, if $A: K \rightarrow$ $H$ is a $\lambda$-inverse strongly monotone with $\lambda \in\left(0, \frac{1}{2}\right]$, then $T:=I-A$ is $(1-2 \lambda)$-strictly pseudocontractive mapping (see [23]).

Lemma 1.8 [27] Let $K$ be a nonempty closed convex subset of a Hilbert space $H$ and $T$ : $K \rightarrow H$ be a $k$-strictly pseudocontractive mapping. Then $I-T$ is demiclosed at zero, that is, whenever $\left\{x_{n}\right\}$ is a sequence in $K$ such that $\left\{x_{n}\right\}$ converges weakly to $x \in K$ and $\left\{(I-T)\left(x_{n}\right)\right\}$ converges strongly to 0 , we have $(I-T)(x)=0$.

From now on, the symbols ' $\rightarrow$ ' and ' $\rightarrow$ ' stand for 'weak convergence' and 'strong convergence', respectively.

Lemma 1.9 Let $H$ be a real Hilbert space and $\left\{x_{n}\right\}$ be a sequence of $H$. If $x_{n} \rightarrow x_{0}$ and $\left\|x_{n}\right\| \rightarrow\left\|x_{0}\right\|$, then $x_{n} \rightarrow x_{0}$.

Lemma 1.10 [28] Let $\left\{a_{n}\right\},\left\{b_{n}\right\},\left\{c_{n}\right\}$ be the sequences of positive numbers satisfying the conditions:

(a) $a_{n+1} \leq\left(1-b_{n}\right) a_{n}+c_{n}$, where $b_{n}<1$;

(b) $\sum_{n=0}^{\infty} b_{n}=+\infty$ and $\lim _{n \rightarrow+\infty} \frac{c_{n}}{b_{n}}=0$.

Then $a_{n} \rightarrow 0$ as $n \rightarrow \infty$.

\section{Regularization}

Through this paper, we will denote by $\mathcal{R}$ and $\mathcal{N}$ the set of all real numbers and the set of natural numbers, respectively. Let $H$ be a real Hilbert space and $K$ be a closed convex subset of $H$. Let $A: H \rightarrow H$ and $A_{i}: K \rightarrow H$ be mappings, where $i=1,2, \ldots, N$. For each $\alpha \in(0,1)$, we now construct a regularization solution $u_{\alpha}$ for the problem (1.2). In fact, we have to solve the following general variational inequality problem: find $u_{\alpha} \in H$ with $g\left(u_{\alpha}\right) \in K$ such that

$$
\left\langle A\left(u_{\alpha}\right)+\alpha^{\mu} \sum_{i=1}^{N}\left(A_{i} \circ g\right)\left(u_{\alpha}\right)+\alpha g\left(u_{\alpha}\right), g(v)-g\left(u_{\alpha}\right)\right\rangle \geq 0
$$

for all $v \in H$ with $g(v) \in K$, where $\mu \in(0,1)$ is a fixed real number.

Theorem 2.1 Let $K$ be a closed convex subset of a real Hilbert space $H$ and $g: H \rightarrow H$ be a mapping such that $K \subset g(H)$. Let $A: H \rightarrow H$ be a hemicontinuous and $g$-monotone mapping. Let $A_{i}$ be a $\lambda_{i}$-inverse strongly monotone mapping of $K$ into $H$ for each $i=1,2, \ldots, N$. If $g$ is an expanding affine continuous mapping and $\mathrm{GVI}_{K}(A, g, S) \neq \emptyset$, then the following conclusions are true: 
(1) For each $\alpha \in(0,1)$, the problem (2.1) has the unique solution $u_{\alpha}$;

(2) $\lim _{\alpha \rightarrow 0^{+}} g\left(u_{\alpha}\right)=g\left(u^{*}\right)$ for some $u^{*} \in \mathrm{GVI}_{K}(A, g, S)$;

(3) There exists a positive constant $M$ such that

$$
\left\|g\left(u_{\alpha}\right)-g\left(u_{\beta}\right)\right\|^{2} \leq \frac{M|\alpha-\beta|}{\alpha^{2}}
$$

for all $\alpha, \beta \in(0,1)$.

Proof Put $\mathcal{I}=\{1,2, \ldots, N\}$. Firstly, due to the definition of an inverse strongly monotone mapping, we may always assume that $\lambda_{i} \in\left(0, \frac{1}{2}\right]$ for each $i \in \mathcal{I}$. Now, for each $i \in \mathcal{I}$, we define functions $F_{0}, F_{i}: g^{-1}(K) \times g^{-1}(K) \rightarrow \mathcal{R}$ by

$$
F_{0}(u, v)=\langle A(u), g(v)-g(u)\rangle, \quad F_{i}(u, v)=\left\langle\left(A_{i} \circ g\right)(u), g(v)-g(u)\right\rangle
$$

for all $(u, v) \in g^{-1}(K) \times g^{-1}(K)$. Note that since $g$ is an affine continuous mapping, we have $g^{-1}(K)$ is a closed convex subset of $H$. Moreover, it is easy to see that $F_{0}$ and $F_{i}$ are monotone bifunctions on $g^{-1}(K)$ for each $i \in \mathcal{I}$.

Now, let $\alpha \in(0,1)$ be a given real number. We construct a function $F_{\alpha}: g^{-1}(K) \times$ $g^{-1}(K) \rightarrow \mathcal{R}$ by

$$
F_{\alpha}(u, v)=F_{0}(u, v)+\alpha^{\mu} \sum_{i=1}^{N} F_{i}(u, v)+\alpha\langle g(u), g(v)-g(u)\rangle
$$

for all $(u, v) \in g^{-1}(K) \times g^{-1}(K)$.

(1) Observe that the problem (2.1) is equivalent to the problem of finding $u_{\alpha} \in g^{-1}(K)$ such that

$$
F_{\alpha}\left(u_{\alpha}, v\right) \geq 0
$$

for all $v \in g^{-1}(K)$. Moreover, one can easily check that $F_{\alpha}(u, v)$ is a monotone hemicontinuous in the variable $u$ for each fixed $v \in g^{-1}(K)$. Indeed, if $g$ is an $\xi$-expanding mapping, then we have $F_{\alpha}(u, v)$ is a strongly monotone mapping with constant $\alpha \xi>0$. Thus, by Lemma 1.4(2), the problem (2.4) has the unique solution $u_{\alpha} \in g^{-1}(K)$ for each $\alpha>0$. This proves (1).

(2) Observe that for each $y \in \operatorname{GVI}_{K}(A, g, S)$, we have $F_{0}\left(y, u_{\alpha}\right) \geq 0$ and $F_{i}\left(y, u_{\alpha}\right)=0$ for each $i \in \mathcal{I}$. This implies that

$$
F_{0}\left(y, u_{\alpha}\right)+\alpha^{\mu} \sum_{i=1}^{N} F_{i}\left(y, u_{\alpha}\right) \geq 0 .
$$

Consequently, by using the monotonicity of $F_{0}$ and $F_{i}$, we see that (2.3), (2.4) and (2.5) imply

$$
\begin{aligned}
0 & \geq-F_{\alpha}\left(u_{\alpha}, y\right) \\
& =-\left[F_{0}\left(u_{\alpha}, y\right)+\alpha^{\mu} \sum_{i=1}^{N} F_{i}\left(u_{\alpha}, y\right)+\alpha\left\langle g\left(u_{\alpha}\right), g(y)-g\left(u_{\alpha}\right)\right\rangle\right]
\end{aligned}
$$




$$
\begin{aligned}
\geq & -F_{0}\left(u_{\alpha}, y\right)-\alpha^{\mu} \sum_{i=1}^{N} F_{i}\left(u_{\alpha}, y\right)-\alpha\left\langle g\left(u_{\alpha}\right), g(y)-g\left(u_{\alpha}\right)\right\rangle \\
& -\left[F_{0}\left(y, u_{\alpha}\right)+\alpha^{\mu} \sum_{i=1}^{N} F_{i}\left(y, u_{\alpha}\right)\right] \\
= & -\left[F_{0}\left(u_{\alpha}, y\right)+F_{0}\left(y, u_{\alpha}\right)\right]-\alpha^{\mu} \sum_{i=1}^{N}\left[F_{i}\left(u_{\alpha}, y\right)+F_{i}\left(y, u_{\alpha}\right)\right]-\alpha\left\langle g\left(u_{\alpha}\right), g(y)-g\left(u_{\alpha}\right)\right\rangle \\
\geq & \alpha\left\langle g\left(u_{\alpha}\right), g\left(u_{\alpha}\right)-g(y)\right\rangle .
\end{aligned}
$$

Hence, we have

$$
\left\langle g\left(u_{\alpha}\right), g(y)-g\left(u_{\alpha}\right)\right\rangle \geq 0
$$

for all $y \in \operatorname{GVI}_{K}(A, g, S)$. This implies

$$
\left\|g\left(u_{\alpha}\right)\right\| \leq\|g(y)\|
$$

for all $y \in \operatorname{GVI}_{K}(A, g, S)$. Thus, $\left\{g\left(u_{\alpha}\right)\right\}$ is a bounded subset of $K$. Consequently, the set of weak limit points as $\alpha \rightarrow 0$ of the net $\left(g\left(u_{\alpha}\right)\right)$, denoted by $\omega_{w}\left(g\left(u_{\alpha}\right)\right)$, is nonempty. This allows us to pick $z \in \omega_{w}\left(g\left(u_{\alpha}\right)\right)$ and a null sequence $\left\{\alpha_{k}\right\}$ in the interval $(0,1)$ such that $\left\{g\left(u_{\alpha_{k}}\right)\right\}$ weakly converges to $z$ as $k \rightarrow \infty$. Notice that since $K$ is closed and convex, we know that $K$ is weakly closed, and so $z \in K$. Consequently, since $K \subset g(H)$, we let $u^{*} \in H$ be such that $z=g\left(u^{\prime \prime}\right)$.

Now, we claim that $u^{*} \in \mathrm{GVI}_{K}(A, g, S)$. To prove this claim, we divide the proof into two steps:

Step 1: We show that $g\left(u^{*}\right) \in S$. Observe that $F_{0}\left(y, u_{\alpha_{k}}\right) \geq 0$ for each $y \in \operatorname{GVI}_{K}(A, g, S)$ and $k \in \mathcal{N}$. Thus, by (2.3) and the monotonicity of $F_{0}$, we see that

$$
\left.\alpha_{k}^{\mu} \sum_{i=1}^{N} F_{i}\left(u_{\alpha_{k}}, y\right)+\alpha_{k} \mid g\left(u_{\alpha_{k}}\right), g(y)-g\left(u_{\alpha_{k}}\right)\right) \geq-F_{0}\left(u_{\alpha_{k}}, y\right) \geq F_{0}\left(y, u_{\alpha_{k}}\right) \geq 0,
$$

that is,

$$
\sum_{i=1}^{N} F_{i}\left(u_{\alpha_{k}}, y\right)+\alpha_{k}^{1-\mu}\left\langle g\left(u_{\alpha_{k}}\right), g(y)-g\left(u_{\alpha_{k}}\right)\right\rangle \geq 0
$$

for any $y \in \operatorname{GVI}_{K}(A, g, S)$ and $k \in \mathcal{N}$. Let $j \in \mathcal{I}$ be given. Pick $y \in \operatorname{GVI}_{K}(A, g, S)$. Since $A_{i}$ is a $\lambda_{i}$-inverse strongly monotone mapping for each $i \in \mathcal{I}$, in view of (2.7), we have

$$
\begin{aligned}
\lambda_{j}\left\|A_{j} \circ g\left(u_{\alpha_{k}}\right)-A_{j} \circ g(y)\right\|^{2} & \leq\left\langle A_{j}\left(g\left(u_{\alpha_{k}}\right)\right)-A_{j}(g(y)), g\left(u_{\alpha_{k}}\right)-g(y)\right\rangle \\
& =\left\langle A_{j}\left(g\left(u_{\alpha_{k}}\right)\right), g\left(u_{\alpha_{k}}\right)-g(y)\right\rangle \\
& \leq \sum_{i=1}^{N}\left\langle A_{i}\left(g\left(u_{\alpha_{k}}\right)\right), g\left(u_{\alpha_{k}}\right)-g(y)\right\rangle \\
& =-\sum_{i=1}^{N} F_{i}\left(u_{\alpha_{k}}, y\right)
\end{aligned}
$$




$$
\begin{aligned}
& \leq \alpha_{k}^{1-\mu}\left\langle g\left(u_{\alpha_{k}}\right), g(y)-g\left(u_{\alpha_{k}}\right)\right\rangle \\
& \leq \alpha_{k}^{1-\mu}\left[\left\|g\left(u_{\alpha_{k}}\right)\right\|\|g(y)\|-\left\|g\left(u_{\alpha_{k}}\right)\right\|^{2}\right] \\
& \leq \alpha_{k}^{1-\mu}\|g(y)\|^{2}
\end{aligned}
$$

for each $k \in \mathcal{N}$. Letting $k \rightarrow \infty$ in (2.9), we obtain

$$
\lim _{k \rightarrow \infty}\left\|A_{j} \circ g\left(u_{\alpha_{k}}\right)\right\|=\lim _{k \rightarrow \infty}\left\|A_{j} \circ g\left(u_{\alpha_{k}}\right)-A_{j} \circ g(y)\right\|=0 .
$$

On the other hand, we know that the mapping $T_{j}:=I-A_{j}$ is strictly pseudocontractive for each $j \in \mathcal{I}$, and hence by Lemma 1.8, we have $A_{j}=I-T_{j}$ is demiclosed at zero. It follows that $A_{j}\left(g\left(u^{*}\right)\right)=0$. This means $g\left(u^{*}\right) \in S_{j}$. Consequently, since $j \in \mathcal{I}$ is arbitrary chosen, we conclude that $g\left(u^{*}\right) \in \bigcap_{i=1}^{N} S_{i}$. This proves Step 1.

Step 2: We show that $u^{*} \in \mathrm{GVI}_{K}(A, g)$. From the monotonic property of $F_{\alpha}$ and (2.4), we have

$$
F_{0}\left(v, u_{\alpha_{k}}\right)+\alpha_{k}^{\mu} \sum_{i=1}^{N} F_{i}\left(v, u_{\alpha_{k}}\right)+\alpha_{k}\left|g(v), g\left(u_{\alpha_{k}}\right)-g(v)\right\rangle=F_{\alpha}\left(v, u_{\alpha_{k}}\right) \leq-F_{\alpha}\left(u_{\alpha_{k}}, v\right) \leq 0
$$

for all $v \in g^{-1}(K)$. This gives

$$
F_{0}\left(v, u_{\alpha_{k}}\right)+\alpha_{k}^{\mu} \sum_{i=1}^{N} F_{i}\left(v, u_{\alpha_{k}}\right) \leq \alpha_{k}\left|g(v), g(v)-g\left(u_{\alpha_{k}}\right)\right\rangle
$$

for all $v \in g^{-1}(K)$. Since the operator $A_{i}$ is a Lipschitzian mapping for each $i \in \mathcal{I}$, it follows that $F_{i}$ is a bounded mapping for each $i \in \mathcal{I}$. Thus, letting $k \rightarrow \infty$, since $\alpha_{k} \rightarrow 0^{+}$and $g\left(u_{\alpha_{k}}\right) \rightarrow g\left(u^{*}\right)$ as $k \rightarrow \infty$, it follows from (2.11) that $F_{0}\left(v, u^{*}\right) \leq 0$ for any $v \in H, g(v) \in K$. Consequently, in view of Lemma 1.4(1), Step 2 is proved.

Hence, from Steps 1 and 2, we conclude that $u^{*} \in \operatorname{GVI}_{K}(A, g, S)$ as required.

Next, we observe that the sequence $\left\{g\left(u_{\alpha_{k}}\right)\right\}$ actually converges strongly to $g\left(u^{*}\right)$. In fact, by using the lower semi-continuity of the norm, we know that

$$
\left\|g\left(u^{*}\right)\right\| \leq \liminf _{k \rightarrow \infty}\left\|g\left(u_{\alpha_{k}}\right)\right\| .
$$

Subsequently, since $u^{*} \in \operatorname{GVI}_{K}(A, g, S)$, we see that (2.7) and (2.12) imply $\left\|g\left(u_{\alpha_{k}}\right)\right\| \rightarrow$ $\left\|g\left(u^{*}\right)\right\|$ as $k \rightarrow \infty$. Then it is straightforward from Lemma 1.9 that the weak convergence to $g\left(u^{*}\right)$ of $\left\{g\left(u_{\alpha_{k}}\right)\right\}$ implies the strong convergence to $g\left(u^{*}\right)$ of $\left\{g\left(u_{\alpha_{k}}\right)\right\}$. Moreover, in view of (2.7), we see that

$$
\left\|g\left(u^{*}\right)\right\|=\inf \left\{\|g(y)\|: y \in \operatorname{GVI}_{K}(A, g, S)\right\} .
$$

Now, we show that

$$
\lim _{\alpha \rightarrow 0^{+}} g\left(u_{\alpha}\right)=g\left(u^{*}\right)
$$

To do so, let $\left\{g\left(u_{\alpha_{j}}\right)\right\} \subset\left(g\left(u_{\alpha}\right)\right)$, where $\left\{\alpha_{j}\right\}$, be any null sequence in the interval $(0,1)$. By following the lines of proof as above (passing to a subsequence if necessary), we know that 
there exists $\tilde{u} \in \operatorname{GVI}_{K}(A, g, S)$ such that $g\left(u_{\alpha_{j}}\right) \rightarrow g(\tilde{u})$ as $j \rightarrow \infty$. Moreover, from (2.7) and (2.13), we have $\|g(\tilde{u})\|=\left\|g\left(u^{*}\right)\right\|$. Consequently, since the function $\|g(\cdot)\|$ is a lower semicontinuous function and $\operatorname{GVI}_{K}(A, g, S)$ is a closed convex subset of $H$, it follows from (2.13) that $u^{*}=\tilde{u}$. This implies that $g\left(u^{*}\right)$ is the strong limit of the net $\left(g\left(u_{\alpha}\right)\right)$ as $\alpha \rightarrow 0^{+}$.

(3) Let $\alpha, \beta \in(0,1)$ and $u_{\alpha}, u_{\beta}$ be the solutions of the problem (2.1) associated with $\alpha$ and $\beta$, respectively. Without loss of generality, we assume that $\alpha<\beta$. For each $i \in \mathcal{I}$, we know that $F_{0}$ and $F_{i}$ are monotone mappings. Then by applying (2.4), we have

$$
\left(\alpha^{\mu}-\beta^{\mu}\right) \sum_{i=1}^{N} F_{i}\left(u_{\alpha}, u_{\beta}\right)+\alpha\left\langle g\left(u_{\alpha}\right), g\left(u_{\beta}\right)-g\left(u_{\alpha}\right)\right\rangle+\beta\left\langle g\left(u_{\beta}\right), g\left(u_{\alpha}\right)-g\left(u_{\beta}\right)\right\rangle \geq 0,
$$

that is,

$$
\left\langle g\left(u_{\alpha}\right)-\frac{\beta}{\alpha} g\left(u_{\beta}\right), g\left(u_{\alpha}\right)-g\left(u_{\beta}\right)\right\rangle \leq \frac{\beta^{\mu}-\alpha^{\mu}}{\alpha} \sum_{i=1}^{N}\left|F_{i}\left(u_{\alpha}, u_{\beta}\right)\right| .
$$

Notice that

$$
\begin{aligned}
& \left\langle g\left(u_{\alpha}\right)-\frac{\beta}{\alpha} g\left(u_{\beta}\right), g\left(u_{\alpha}\right)-g\left(u_{\beta}\right)\right\rangle \\
& \quad=\left\|g\left(u_{\alpha}\right)-g\left(u_{\beta}\right)\right\|^{2}+\frac{\alpha-\beta}{\alpha}\left\langle g\left(u_{\beta}\right), g\left(u_{\alpha}\right)\right\rangle-\frac{\alpha-\beta}{\alpha}\left\|g\left(u_{\beta}\right)\right\|^{2} \\
& \quad \geq\left\|g\left(u_{\alpha}\right)-g\left(u_{\beta}\right)\right\|^{2}+\frac{\alpha-\beta}{\alpha}\left\langle g\left(u_{\beta}\right), g\left(u_{\alpha}\right)\right\rangle
\end{aligned}
$$

since $0<\alpha<\beta$. Using this one together with (2.14), we have

$$
\left\|g\left(u_{\alpha}\right)-g\left(u_{\beta}\right)\right\|^{2} \leq \frac{\beta-\alpha}{\alpha} \theta^{2}+\frac{\beta^{\mu}-\alpha^{\mu}}{\alpha} \sum_{i=1}^{N}\left|F_{i}\left(u_{\alpha}, u_{\beta}\right)\right|,
$$

where $\theta=\sup \left\{\left\|g\left(u_{\alpha}\right)\right\|: \alpha \in(0,1)\right\}$. Consequently, by using the boundedness of $F_{i}$, we have

$$
\left\|g\left(u_{\alpha}\right)-g\left(u_{\beta}\right)\right\|^{2} \leq \frac{\beta-\alpha}{\alpha} \theta^{2}+\frac{\beta^{\mu}-\alpha^{\mu}}{\alpha} M_{2}
$$

for some $M_{2}>0$.

Finally, by applying Lagrange's mean-value theorem to a function which is defined on $[1,+\infty)$ by $t \mapsto t^{-\mu}$, we see that

$$
\left\|g\left(u_{\alpha}\right)-g\left(u_{\beta}\right)\right\|^{2} \leq \frac{\beta-\alpha}{\alpha^{2}} \theta^{2}+\frac{\beta-\alpha}{\alpha^{2}} \cdot \mu M_{2} \leq \frac{\beta-\alpha}{\alpha^{2}} M
$$

where $M=\max \left\{\theta^{2}, \mu M_{2}\right\}$. This completes the proof.

Remark 2.2 As a special case of Theorem 2.1, if $g$ is the identity operator on $H$, then we recover a recent result presented by Kim and Buong [24]. 


\section{Main results}

In this section, we provide a regularization inertial proximal point algorithm for finding a solution of the problem (1.2). In fact, starting with an element $z_{1} \in H$ such that $g\left(z_{1}\right) \in K$, we consider the following processes:

$$
\left\langle c_{n}\left[A\left(z_{n+1}\right)+\alpha_{n}^{\mu} \sum_{i=1}^{N}\left(A_{i} \circ g\right)\left(z_{n+1}\right)+\alpha_{n} g\left(z_{n+1}\right)\right]+g\left(z_{n+1}\right)-g\left(z_{n}\right), g(v)-g\left(z_{n+1}\right)\right\rangle \geq 0
$$

for all $v \in H, g(v) \in K$, where $\left\{c_{n}\right\}$ and $\left\{\alpha_{n}\right\}$ are sequences of positive real numbers.

The well-definedness of algorithm (3.1) is guaranteed by the following proposition:

Proposition 3.1 Assume that all hypotheses of Theorem 2.1 are satisfied. Let $z \in g^{-1}(K)$ be a given element and $c, \alpha$ be positive real numbers. Define a bifunction $F_{(z, c, \alpha)}: g^{-1}(K) \times$ $g^{-1}(K) \rightarrow \mathcal{R}$ by

$$
F_{(z, c, \alpha)}(u, v):=\left\langle c\left[A(u)+\alpha^{\mu} \sum_{i=1}^{N}\left(A_{i} \circ g\right)(u)+\alpha g(u)\right]+g(u)-g(z), g(v)-g(u)\right\rangle
$$

for all $u, v \in g^{-1}(K)$. Then there exists a unique element $u^{*} \in g^{-1}(K)$ such that $F_{(z, c, \alpha)}\left(u^{*}, v\right) \geq$ 0 for all $v \in g^{-1}(K)$.

Proof Assume that $g$ is an $\xi$-expanding mapping. Then, for any $u, v \in g^{-1}(K)$, we see that

$$
\begin{aligned}
F_{(z, c, \alpha)}(u, v)+F_{(z, c, \alpha)}(v, u) & \leq(1+c \alpha)\langle g(u)-g(v), g(v)-g(u)\rangle \\
& =-(1+c \alpha)\|g(u)-g(v)\|^{2} \\
& \leq-\xi(1+c \alpha)\|u-v\|^{2} .
\end{aligned}
$$

This means $F_{(z, c, \alpha)}$ is $\xi(1+c \alpha)$-strongly monotone. Consequently, by Lemma 1.4(2), the proof is completed.

Now, we provide some sufficient conditions for the convergence of the regularization inertial proximal point algorithm (3.1).

Theorem 3.2 Assume that all hypotheses of Theorem 2.1 are satisfied. If the parameters $c_{n}$ and $\alpha_{n}$ are positive real numbers such that

(a) $\lim _{n \rightarrow \infty} \alpha_{n}=0$;

(b) $\lim _{n \rightarrow \infty} \frac{\alpha_{n}-\alpha_{n+1}}{\alpha_{n+1}^{2}}=0$;

(c) $\liminf _{n \rightarrow \infty} c_{n} \alpha_{n}>0$,

then the sequence $\left\{g\left(z_{n}\right)\right\}$ defined by (3.1) converges strongly to an element $g\left(u^{*}\right) \in H$ for some $u^{*} \in \operatorname{GVI}_{K}(A, g, S)$.

Proof Let $v \in H$ such that $g(v) \in K$. From (3.1), for each $n \in \mathcal{N}$, we see that

$$
\left\langle c_{n}\left[A\left(z_{n+1}\right)+\alpha_{n}^{\mu} \sum_{i=1}^{N}\left(A_{i} \circ g\right)\left(z_{n+1}\right)\right]+\left(1+c_{n} \alpha_{n}\right) g\left(z_{n+1}\right)-g\left(z_{n}\right), g(v)-g\left(z_{n+1}\right)\right\rangle \geq 0,
$$


that is,

$$
\begin{aligned}
& \left\langle c_{n}\left[A\left(z_{n+1}\right)+\alpha_{n}^{\mu} \sum_{i=1}^{N}\left(A_{i} \circ g\right)\left(z_{n+1}\right)\right]+\left(1+c_{n} \alpha_{n}\right) g\left(z_{n+1}\right), g(v)-g\left(z_{n+1}\right)\right\rangle \\
& \quad \geq\left\langle g\left(z_{n}\right), g(v)-g\left(z_{n+1}\right)\right\rangle .
\end{aligned}
$$

Thus we have

$$
\begin{aligned}
& \left(1+c_{n} \alpha_{n}\right)\left\langle\frac{c_{n}}{\left(1+c_{n} \alpha_{n}\right)}\left[A\left(z_{n+1}\right)+\alpha_{n}^{\mu} \sum_{i=1}^{N}\left(A_{i} \circ g\right)\left(z_{n+1}\right)\right]+g\left(z_{n+1}\right), g(v)-g\left(z_{n+1}\right)\right\rangle \\
& \geq\left\{g\left(z_{n}\right), g(v)-g\left(z_{n+1}\right)\right\rangle
\end{aligned}
$$

and so

$$
\begin{aligned}
& \left\langle\frac{c_{n}}{\left(1+c_{n} \alpha_{n}\right)}\left[A\left(z_{n+1}\right)+\alpha_{n}^{\mu} \sum_{i=1}^{N}\left(A_{i} \circ g\right)\left(z_{n+1}\right)\right]+g\left(z_{n+1}\right), g(v)-g\left(z_{n+1}\right)\right\rangle \\
& \quad \geq \frac{1}{\left(1+c_{n} \alpha_{n}\right)}\left\langle g\left(z_{n}\right), g(v)-g\left(z_{n+1}\right)\right\rangle .
\end{aligned}
$$

Hence we have

$$
\left\langle\gamma_{n}\left[A\left(z_{n+1}\right)+\alpha_{n}^{\mu} \sum_{i=1}^{N}\left(A_{i} \circ g\right)\left(z_{n+1}\right)\right]+g\left(z_{n+1}\right), g(v)-g\left(z_{n+1}\right)\right\rangle \geq \beta_{n}\left\langle g\left(z_{n}\right), g(v)-g\left(z_{n+1}\right)\right\rangle
$$

for any $v \in H$ with $g(v) \in K$ and $n \in \mathcal{N}$, where

$$
\beta_{n}=\frac{1}{\left(1+c_{n} \alpha_{n}\right)}, \quad \gamma_{n}=c_{n} \beta_{n}
$$

By the similar argument, it follows from (2.1) that

$$
\left\langle c_{n} A\left(u_{n}\right)+\alpha_{n}^{\mu} \sum_{i=1}^{N}\left(A_{i} \circ g\right)\left(u_{n}\right)+\alpha_{n} g\left(u_{n}\right)+g\left(u_{n}\right)-g\left(u_{n}\right), g(v)-g\left(u_{n}\right)\right\rangle \geq 0
$$

and so

$$
\begin{aligned}
& \left\langle\frac{c_{n}}{\left(1+c_{n} \alpha_{n}\right)}\left[A\left(u_{n}\right)+\alpha_{n}^{\mu} \sum_{i=1}^{N}\left(A_{i} \circ g\right)\left(u_{n}\right)\right]+g\left(u_{n}\right), g(v)-g\left(u_{n}\right)\right\rangle \\
& \quad \geq \frac{1}{\left(1+c_{n} \alpha_{n}\right)}\left\langle g\left(u_{n}\right), g(v)-g\left(u_{n}\right)\right\rangle,
\end{aligned}
$$

when $u_{n}$ is the solution of (2.1) and $\alpha$ is replaced by $\alpha_{n}$. Thus we have

$$
\left\langle\gamma_{n}\left[A\left(u_{n}\right)+\alpha_{n}^{\mu} \sum_{i=1}^{N}\left(A_{i} \circ g\right)\left(u_{n}\right)\right]+g\left(u_{n}\right), g(v)-g\left(u_{n}\right)\right\rangle \geq \beta_{n}\left\langle g\left(u_{n}\right), g(v)-g\left(u_{n}\right)\right\rangle,
$$


for any $v \in H$ with $g(v) \in K$ and $n \in \mathcal{N}$. By setting $v=u_{n}$ in (3.2), we have

$$
\left\langle\gamma_{n}\left[A\left(z_{n+1}\right)+\alpha_{n}^{\mu} \sum_{i=1}^{N}\left(A_{i} \circ g\right)\left(z_{n+1}\right)\right]+g\left(z_{n+1}\right), g\left(u_{n}\right)-g\left(z_{n+1}\right)\right\rangle \geq \beta_{n}\left|g\left(z_{n}\right), g\left(u_{n}\right)-g\left(z_{n+1}\right)\right\rangle
$$

and, by setting $v=z_{n+1}$ in (3.3), we have

$$
\left\langle\gamma_{n}\left[A\left(u_{n}\right)+\alpha_{n}^{\mu} \sum_{i=1}^{N}\left(A_{i} \circ g\right)\left(u_{n}\right)\right]+g\left(u_{n}\right), g\left(z_{n+1}\right)-g\left(u_{n}\right)\right\rangle \geq \beta_{n}\left\langle g\left(u_{n}\right), g\left(z_{n+1}\right)-g\left(u_{n}\right)\right\rangle .
$$

Thus, by adding two inequalities above, we have

$$
\begin{aligned}
\gamma_{n}\left\langle A\left(z_{n+1}\right)-A\left(u_{n}\right)+\alpha_{n}^{\mu} \sum_{i=1}^{N}\left[A_{i} \circ g\left(z_{n+1}\right)-A_{i} \circ g\left(u_{n}\right)\right], g\left(u_{n}\right)-g\left(z_{n+1}\right)\right\rangle \\
\quad+\left\langle g\left(z_{n+1}\right)-g\left(u_{n}\right), g\left(u_{n}\right)-g\left(z_{n+1}\right) t\right\rangle \\
\geq \beta_{n}\left\langle g\left(z_{n}\right)-g\left(u_{n}\right), g\left(u_{n}\right)-g\left(z_{n+1}\right)\right\rangle .
\end{aligned}
$$

Notice that since $A$ is a $g$-monotone mapping and each $A_{i}$ for each $i \in \mathcal{I}$ is a $\lambda_{i}$-inverse strongly monotone, we have

$$
\left\langle A\left(z_{n+1}\right)-A\left(u_{n}\right), g\left(u_{n}\right)-g\left(z_{n+1}\right)\right\rangle \leq 0
$$

and

$$
\left\langle A_{i} \circ g\left(z_{n+1}\right)-A_{i} \circ g\left(u_{n}\right), g\left(u_{n}\right)-g\left(z_{n+1}\right)\right\rangle \leq 0
$$

for each $i \in \mathcal{I}$. Thus, from the above fact, we obtain

$$
\left\langle g\left(z_{n+1}\right)-g\left(u_{n}\right), g\left(u_{n}\right)-g\left(z_{n+1}\right)\right\rangle \geq \beta_{n}\left|g\left(z_{n}\right)-g\left(u_{n}\right), g\left(u_{n}\right)-g\left(z_{n+1}\right)\right\rangle .
$$

This gives

$$
\left\|g\left(z_{n+1}\right)-g\left(u_{n}\right)\right\| \leq \beta_{n}\left\|g\left(z_{n}\right)-g\left(u_{n}\right)\right\|
$$

for each $n \in \mathcal{N}$. Thus, using Theorem 2.1(3), we have

$$
\begin{aligned}
\left\|g\left(z_{n+1}\right)-g\left(u_{n+1}\right)\right\| & \leq\left\|g\left(z_{n+1}\right)-g\left(u_{n}\right)\right\|+\left\|g\left(u_{n}\right)-g\left(u_{n+1}\right)\right\| \\
& \leq \beta_{n}\left\|g\left(z_{n}\right)-g\left(u_{n}\right)\right\|+\sqrt{\frac{M\left(\alpha_{n}-\alpha_{n+1}\right)}{\alpha_{n+1}^{2}}} \\
& \leq\left(1-b_{n}\right)\left\|g\left(z_{n}\right)-g\left(u_{n}\right)\right\|+d_{n},
\end{aligned}
$$

where

$$
b_{n}=\frac{c_{n} \alpha_{n}}{\left(1+c_{n} \alpha_{n}\right)}, \quad d_{n}=\sqrt{\frac{M\left(\alpha_{n}-\alpha_{n+1}\right)}{\alpha_{n+1}^{2}}} .
$$


Consequently, by the condition (c), we have $\sum_{n=1}^{\infty} b_{n}=\infty$. Meanwhile, the conditions (b) and (c) imply $\lim _{n \rightarrow \infty} \frac{d_{n}}{b_{n}}=0$. Thus, all the conditions of Lemma 1.10 are satisfied, and so $\left\|g\left(z_{n+1}\right)-g\left(u_{n+1}\right)\right\| \rightarrow 0$ as $n \rightarrow \infty$. Moreover, by the condition (a) and Theorem 2.1(2), we know that there exists $u^{*} \in \operatorname{GVI}_{K}(A, g, S)$ such that $\left\{g\left(u_{n}\right)\right\}$ converges strongly to $g\left(u^{*}\right)$. This implies that $\left\{g\left(z_{n}\right)\right\}$ converges strongly to $g\left(u^{*}\right)$ as $n \rightarrow+\infty$. This completes the proof.

Example 3.3 Let $p \in(0,1)$ be a fixed real number. Let $\alpha_{n}=\left(\frac{1}{n}\right)^{p}$ and $c_{n}=\frac{1}{\alpha_{n}}$ for each $n \in \mathcal{N}$. Then we can check that the sequences $\left\{\alpha_{n}\right\}$ and $\left\{c_{n}\right\}$ satisfy all the conditions in Theorem 3.2.

Remark 3.4 Note that because of the condition (b) of Theorem 3.2, the choice $\left\{\frac{1}{n}\right\}$ is not included in the class of the parameters $\left\{\alpha_{n}\right\}$. This may lead to an important further research work, that is, finding other (regularization inertial proximal point) algorithms for the problem (1.2) including the natural parameter choice $\left\{\frac{1}{n}\right\}$.

\section{Conclusion}

In this paper, we provide a regularization method for general monotone variational inequality, where the regularizer is a Lipschitz continuous and strongly monotone operator. Also, an iterative method as discretization of regularization method is introduced. We would like to point out, as we have mentioned in Remark 1.1 and Remark 1.2, that our results are general and extend some well-known results which have been investigated in literature; for more examples, see [29-34]. Further, let us notice that although many authors have proved results for finding the solution of the variational inequality problem and the solution set of a finite inverse strongly monotone mapping, it is clear that it cannot be directly applied to our main considered problem (1.2) due to the presence of $g$.

Competing interests

The authors declare that they have no competing interests.

Authors' contributions

Both authors contributed equally in this paper. They read and approved the final manuscript.

\section{Author details}

${ }^{1}$ Department of Mathematics Education and the RINS, Gyeongsang National University, Chinju, 660-701, Korea.

${ }^{2}$ Department of Mathematics, Faculty of Science, Naresuan University, Phitsanulok, 65000, Thailand. ${ }^{3}$ The Centre of Excellence in Mathematics, CHE, Si Ayuthaya Road, Bangkok, 10400, Thailand.

\footnotetext{
Acknowledgements

The authors wish to express their gratitude to the referees for a careful reading of the manuscript and helpful suggestions. This work is supported by the Centre of Excellence in Mathematics, the commission on Higher Education, Thailand and by the Basic Science Research Program through the National Research Foundation of Korea funded by the Ministry of Education, Science and Technology (Grant Number: 2010-0021821)

Received: 8 May 2012 Accepted: 13 September 2012 Published: 3 October 2012

References

1. Hartman, P, Stampacchia, G: On some nonlinear elliptic differential functional equations. Acta Math. 115, 271-310 (1966)

2. Noor, MA: General variational inequalities. Appl. Math. Lett. 1, 119-121 (1988)

3. Agarwal, RP, Cho, YJ, Petrot, N: Systems of general nonlinear set-valued mixed variational inequalities problems in Hilbert spaces. Fixed Point Theory Appl. 2011, 31 (2011)

4. Cho, YJ, Argyros, IK, Petrot, N: Approximation methods for common solutions of generalized equilibrium, systems of nonlinear variational inequalities and fixed point problems. Comput. Math. Appl. 60, 2292-2301 (2010)

5. Cho, YJ, Kang, SM, Qin, X: On systems of generalized nonlinear variational inequalities in Banach spaces. Appl. Math Comput. 206, 214-220 (2008)

6. Cho, YJ, Kim, JK, Verma, RU: A class of nonlinear variational inequalities involving partially relaxed monotone mappings and general auxiliary principle. Dyn. Syst. Appl. 11, 333-338 (2002)
} 
7. Cho, YJ, Qin, X: Systems of generalized nonlinear variational inequalities and its projection methods. Nonlinear Anal. 69, 4443-4451 (2008)

8. Cho, YJ, Qin, X: Generalized systems for relaxed cocoercive variational inequalities and projection methods in Hilbert spaces. Math. Inequal. Appl. 12, 365-375 (2009)

9. Noor, MA: Some developments in general variational inequalities. Appl. Math. Comput. 152, 199-277 (2004)

10. Yao, Y, Cho, YJ, Chen, RD: An iterative algorithm for solving fixed point problems, variational inequality problems and mixed equilibrium problems. Nonlinear Anal. 71, 3363-3373 (2009)

11. Yao, Y, Cho, YJ, Liou, Y: Hierarchical convergence of an implicit double-net algorithm for nonexpansive semigroups and variational inequality problems. Fixed Point Theory Appl. 2011, 101 (2011)

12. Yao, Y, Cho, YJ, Liou, Y: Iterative algorithms for variational inclusions, mixed equilibrium problems and fixed point problems approach to optimization problems. Cent. Eur. J. Math. 9, 640-656 (2011)

13. Petrot, N: Existence and algorithm of solutions for general set-valued Noor variational inequalities with relaxed $(\mu, v)$-cocoercive operators in Hilbert spaces. J. Appl. Math. Comput. 32, 393-404 (2010)

14. Halpern, B: Fixed points of nonexpansive maps. Bull. Am. Math. Soc. 73, 957-961 (1967)

15. Lau, AT, Miyake, H, Takahashi, W: Approximation of fixed points for amenable semigroups of nonexpansive mappings in Banach spaces. Nonlinear Anal. 67, 1211-1225 (2007)

16. Moudafi, A: Viscosity approximation methods for fixed-points problems. J. Math. Anal. Appl. 12, 46-55 (2000)

17. Suzuki, T: Strong convergence of Kranoselskii and Mann type sequences for one-parameter nonexpansive semigroups without Bochner integrals. J. Math. Anal. Appl. 305, 227-239 (2005)

18. Chang, SS, Lee, HWJ, Chan, CK: A new method for solving equilibrium problem fixed point problem and variational inequality problem with application to optimization. Nonlinear Anal. 70, 3307-3319 (2009)

19. Inchan, I, Petrot, N: System of general variational inequalities involving different nonlinear operators related to fixed point problems and its applications. Fixed Point Theory Appl. 2011, Article ID 689478 (2011)

20. Nadezhkina, N, Takahashi, W: Weak convergence theorem by an extragradient method for nonexpansive mappings and monotone mappings. J. Optim. Theory Appl. 128, 191-201 (2006)

21. Petrot, N: A resolvent operator technique for approximate solving of generalized system mixed variational inequality and fixed point problems. Appl. Math. Lett. 23, 440-445 (2010)

22. Cho, YJ, Petrot, N: Regularization and iterative method for general variational inequality problem in Hilbert spaces. J. Inequal. Appl. 2011, 21 (2011)

23. liduka, H, Takahashi, W: Strong convergence theorems for nonexpansive mappings and inverse-strongly monotone mappings. Nonlinear Anal. 61, 341-350 (2005)

24. Kim, JK, Buong, N: Regularization inertial proximal point algorithm for monotone hemicontinuous mapping and inverse strongly monotone mappings in Hilbert spaces. J. Inequal. Appl. 2010, Article ID 451916 (2010)

25. Takahashi, W, Toyoda, M: Weak convergence theorems for nonexpansive mappings and monotone mappings. J. Optim. Theory Appl. 118, 417-428 (2003)

26. Blum, E, Oettli, W: From optimization and variational inequalities to equilibrium problems. Math. Stud. 63, 123-145 (1994)

27. Osilike, MO, Udomene, A: Demiclosedness principle and convergence theorems for strictly pseudocontractive mappings of Browder-Petryshyn type. J. Math. Anal. Appl. 256, 431-445 (2001)

28. Xu, HK: Iterative algorithms for nonlinear operators. J. Lond. Math. Soc. 66, 240-256 (2002)

29. Ceng, L-C, Ansari, QH, Yao, JC: Mann-type steepest-descent and modified hybrid steepest-descent methods for variational inequalities in Banach spaces. Numer. Funct. Anal. Optim. 29(9-10), 987-1033 (2008)

30. Chen, $R, S u, Y, X u, H K$ : Regularization and iteration methods for a class of monotone variational inequalities. Taiwan. J. Math. 13(2B), 739-752 (2009)

31. Lu, X, Xu, HK, Yin, X: Hybrid methods for a class of monotone variational inequalities. Nonlinear Anal., Theory Methods Appl. 71(3-4), 1032-1041 (2009)

32. Siddiqi, AH, Ansari, QH: General strongly nonlinear variational inequalities. J. Math. Anal. Appl. 166(2), 386-392 (1992)

33. Siddiqi, AH, Ansari, QH, Kazmi, KR: On nonlinear variational inequalities. Indian J. Pure Appl. Math. 25(9), 969-973 (1994)

34. Yao, JC: Variational inequalities with generalized monotone operators. Math. Oper. Res. 19(3), 691-705 (1994)

doi:10.1186/1687-1812-2012-169

Cite this article as: Cho and Petrot: Regularization method for Noor's variational inequality problem induced by a hemicontinuous monotone operator. Fixed Point Theory and Applications 2012 2012:169.

\section{Submit your manuscript to a SpringerOpen ${ }^{0}$ journal and benefit from:}

- Convenient online submission

- Rigorous peer review

- Immediate publication on acceptance

Open access: articles freely available online

- High visibility within the field

- Retaining the copyright to your article 